\section{REVISTA BRASILEIRA DE QUALIDADE DE VIDA}

\title{
Efeito de um programa linear de treinamento resistido sobre a autonomia funcional, a flexibilidade, a força e a qualidade de vida de mulheres em idade avançada
}

\section{Effect of a linear resistance training program on the functional autonomy, the flexibility, the strength and the quality of life of older women}

\author{
Weverton Taynan Lima da Silva \\ Universidade do Estado do Pará - UEPA - Campus de Tucuruí - Tucuruí - Pará - Brasil \\ weverton_taynan@hotmail.com \\ Claudio Joaquim Borba-Pinheiro \\ Universidade do Estado do Pará - UEPA - Campus de Tucuruí - Tucuruí - Pará - Brasil \\ Instituto Federal do Pará - IFPA - Campus de Tucuruí - Tucuruí - Pará - Brasil \\ Laboratório de Biociências da Motricidade Humana - Universidade Tiradentes - UNIT - Aracajú - Sergipe - Brasil \\ claudioborba18@gmail.com
}

\section{RESUMO}

OBJETIVO: Verificar os efeitos de um programa de exercícios resistidos (ER) na força muscular, na flexibilidade, na autonomia funcional e na qualidade de vida (QV) de mulheres com idade avançada.

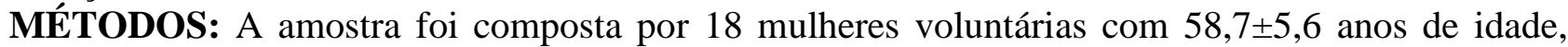
$69 \pm 14,5 \mathrm{~kg}$ de massa corporal, $1,53 \pm 0,06 \mathrm{~m}$ de estatura e $29,2 \pm 4,2 \mathrm{~kg} / \mathrm{m}^{2}$ de Índice de Massa Corporal (IMC) residentes no município de Tucuruí/PA. O treinamento foi periodizado em seis meses, divididos em três ciclos bimestrais, com intensidade progressiva de $50-70 \%$ de uma repetição máxima (1RM). A estatística foi realizada pelo teste ANOVA one way com Post-Hoc de Bonferroni para os testes de força e o teste $T$ Student para as demais variáveis.

RESULTADOS: Os dados apontam melhoras significativas $(\mathrm{p}<0,05)$ do grupo para todas as medidas de força dos membros superiores e inferiores, como bíceps cross $(\Delta \%=40,5 \%)$ e leg press horizontal $(\Delta \%=31,0 \%)$, assim como apresentou melhoras $(\mathrm{p}<0,05)$ na flexibilidade $(\Delta \%=76,8 \%)$. Além disso, a autonomia funcional $(\Delta \%=-10,4 \%)$ e a QV $(\Delta \%=17,9 \%)$ também apresentaram melhoras $(\mathrm{p}<0,05)$.

CONCLUSÃO: O método de ER utilizado foi efetivo para melhorar a flexibilidade, a força muscular, a autonomia funcional e a QV de mulheres com idade avançada.

PALAVRAS-CHAVE: Envelhecimento. Treinamento resistido. Autonomia Funcional. Qualidade de vida. 


\section{ABSTRACT}

OBJECTIVE: To determine the effects of resistance training (RT) on muscle strength, flexibility, functional autonomy and quality of life (QoL) in older women.

METHODS: The sample consisted of 18 female volunteers with $58.7 \pm 5.6$ years-old, $69 \pm 14.5$ $(\mathrm{kg})$ body mass, $1.53 \pm 0.06(\mathrm{~m})$ in height and $29.2 \pm 4.2\left(\mathrm{~kg} / \mathrm{m}^{2}\right)$ of BMI residents in the municipal Tucuruí-State Pará. The RT was periodized in six months, divided into three bimonthly cycles, with progressive intensity of 50-70\% of 1RM. Statistical testing was performed by one-way ANOVA with Bonferroni post-hoc tests for strength and the Student T test for the other variables.

RESULTS: The data show significant improvements $(\mathrm{p}<0.05)$ in the group for all measures in strength of arms and legs as: biceps cross $(\Delta \%=40.5 \%)$ and horizontal leg press $(\Delta \%=31 \%)$; and also showed improvement $(\mathrm{p}<0.05)$ for flexibility $(\Delta \%=76.8 \%)$.In addition, the functional autonomy GDLAM index (IG) $(\Delta \%=-10.4 \%)$ and QoL $(\Delta \%=17,9 \%)$ also showed improvements $(\mathrm{p}<0.05)$.

CONCLUSION: The RT method was effective to improve flexibility, muscle strength, functional autonomy and QoL in women with advanced age in this study.

KEYWORDS: Aging. Resistance training. Functional autonomy. Quality of life.

\section{Introdução}

Nos últimos anos aumentou o número de pessoas que atingem a idade idosa e, consequentemente, a expectativa de vida da população. A projeção para 2050 é que haverá mais idosos que crianças menores de 15 anos. Em 2012, foi constatado que as pessoas com 60 anos ou mais atingiram 810 milhões, constituindo 11,5\% da população global. Projeta-se que esse número alcance 1 bilhão em menos de 10 anos e, mais, que duplique até 2050. No Brasil, em 1991 somavam-se 10,7 milhões de idosos e, esse número, em 2011, saltou para 23,5 milhões de pessoas acima dos 60 anos (BRASIL, 2013).

No entanto, isso não garante que as pessoas que estão vivendo mais, estão vivendo melhor (BLOCK et al., 2008). Essa problemática chama a atenção da comunidade científica. No período 1982-2010, houve um aumento considerável no número de trabalhos científicos voltados ao envelhecimento humano na América Latina e no Brasil, onde, dentre outros assuntos, os mais abordados foram: 51,0\% para o estado de saúde e 17,9\% para atividade física e saúde (BEZERRA; ALMEIDA; NOBREGA-THERRIEN, 2012).

$\mathrm{O}$ processo de envelhecimento provoca alterações anatômicas e fisiológicas que afetam negativamente a capacidade funcional e a qualidade de vida (QV) (GAEDE-CARRILLO et al., 2015). Essas alterações se acentuam devido à insuficiente atividade do sistema neuromuscular, que é consequência de maus hábitos de estilo de vida que incluem a inatividade física (REBELATTO et al., 2006). Para os mesmos autores, o decréscimo do sistema neuromuscular evidencia-se através da sarcopenia, que é a perda de massa muscular, causadora da debilidade no referido sistema, dentre elas, a redução da força muscular e da flexibilidade, com implicações na capacidade de coordenação e de controle do equilíbrio corporal (REBELATTO et al., 2006).

Diante disto, observa-se que o exercício físico assume um papel fundamental no controle e na melhora dos fatores de morbidade, pois, conforme o idoso é estimulado a praticar exercícios regulares, pode evitar maiores perdas na capacidade funcional e na QV (AMORIN; DANTAS, 2002; ACSM, 2014; GAEDE-CARRILLO et al., 2015). Idosos praticantes de atividades físicas não só aumentam a força muscular como, também, o equilíbrio e a mobilidade, reduzindo os riscos de lesões causadas por acidentes domésticos e criando uma maior autonomia funcional (PEREIRAJUNIOR et al., 2010; CABRAL et al., 2014). 
Dentre vários exercícios físicos, um que se destaca na literatura é o exercício resistido (ER), por promover vários benefícios aos idosos, como: diminuição de dores musculares, aumento da massa óssea, aumento da força muscular, melhora do equilíbrio, aumento ou manutenção da flexibilidade, melhora da QV e da autonomia funcional (BORBA-PINHEIRO et al., 2010; TEIXEIRA; HESPANHOL; MARQUEZ, 2012; CABRAL et al., 2014; FARIAS et al., 2014).

Nesse contexto, o presente estudo teve como objetivo verificar os efeitos de um programa de ER na força muscular, na flexibilidade, na autonomia funcional e na QV de mulheres com idade avançada.

\section{Métodos}

Este estudo foi caracterizado como uma pesquisa experimental com delineamento préexperimental (THOMAS; NELSON; SILVERMAN, 2007).

Os critérios de inclusão na amostra foram: idade igual ou superior a 50 anos, que não participaram de qualquer programa de exercício físico nos seis meses que precederam o início do estudo e com ausência de qualquer doença ou distúrbio que pudesse influenciar nos resultados da pesquisa. Foram excluídos do estudo indivíduos que não completaram o tempo de intervenção e/ou não completaram as avaliações das variáveis deste estudo. Ao fim da seleção, a amostra foi composta por 18 mulheres residentes no município de Tucuruí-PA, submetidas a um programa de ER com duração de 6 meses, no ano de 2014.

As voluntárias assinaram o termo de consentimento livre e esclarecido, atendendo as orientações da resolução 466/12 do Conselho Nacional de Saúde, de dezembro de 2012, sobre experimentos com humanos (BRASIL, 2012). A pesquisa foi aprovada pelo comitê de ética da Universidade Federal do Rio de Janeiro, protocolo n ${ }^{\circ}$ 0050/2011.

Para definir as características da amostra foi realizada a avaliação dos parâmetros antropométricos seguindo recomendação da Organização Mundial da Saúde (WORLD HEALTH ORGANIZATION, 1995). Utilizando as medidas de massa corporal e estatura determinadas em uma balança antropométrica Welmy® $\mathrm{CH} 110$ (Brasil), com capacidade máxima de $150 \mathrm{~kg}$ e resolução de $100 \mathrm{~g}$, e estadiômetro vertical fixo à balança para medir a estatura das participantes, sendo o resultado expresso em cm, com resolução de $0,1 \mathrm{~cm}$. A partir dos dados de massa corporal e estatura, foi feito o cálculo do Índice de Massa Corporal (IMC), seguindo a fórmula:

$$
\left[\mathrm{IMC}=\operatorname{Massa}(\mathrm{kg}) / \text { Estatura }(\mathrm{m})^{2}\right]
$$

Para a avaliação da autonomia funcional das voluntárias, optou-se por utilizar os testes do protocolo de avaliação da autonomia funcional do Grupo de Desenvolvimento Latino-Americano para a maturidade (GDLAM) (DANTAS et al., 2014). O protocolo é constituído de cinco testes, todos aferidos por tempo em s e se relacionam com os principais movimentos da vida diária do idoso. Tais testes são:

a) Caminhar $10 \mathrm{~m}(\mathrm{C} 10 \mathrm{~m})$ - o teste avalia a velocidade que o indivíduo leva para percorrer 10m;

b) Levantar-se da posição sentada (LPS) - avalia a capacidade funcional da extremidade inferior;

c) Levantar-se da posição decúbito ventral (LPDV) - avalia a habilidade do indivíduo em se levantar do chão;

d) Levantar-se da cadeira e locomover-se pela casa (LCLC) - avalia a capacidade do idoso na sua agilidade e equilíbrio, em situações de vida;

e) Teste de vestir e tirar a camisa (VTC) - serve para avaliar a autonomia funcional dos membros superiores. 
O índice GDLAM (IG) é calculado pela seguinte fórmula:

$$
\mathrm{IG}=[(\mathrm{C} 10 \mathrm{~m}+\mathrm{LPS}+\mathrm{LPDV}+\mathrm{VTC}) * 2)+\mathrm{LCLC} / 4]
$$

Para coletar os dados relacionados à QV, foi utilizado o questionário SF-36, no qual diferentes aspectos são avaliados:

a) Componentes de saúde física: capacidade funcional, aspectos físicos, aspectos emocionais, intensidade de dor, estado geral da saúde;

b) Componentes de saúde mental: vitalidade, aspectos sociais e saúde mental.

Todos os dados referentes aos aspectos geram os escores de Saúde Física; Saúde Mental e Escore Total de QV (CICONELLI et al., 1999).

Utilizou-se para avaliação da força muscular o teste de uma repetição máxima (1RM). Antes do teste de 1RM foi solicitado que as voluntárias realizassem um breve aquecimento específico, permitindo a execução de uma série de, no máximo, 20 repetições com cargas leves (RAMALHO et al., 2011). A carga máxima considerada para 1RM do indivíduo foi aquela em que a execução seguiu um padrão biomecânico adequado de execução técnica do movimento. Em casos onde a carga máxima não foi encontrada em seis tentativas, a voluntaria foi submetia a um novo teste, realizado após 48 horas do teste anterior (ACSM, 2014; RAMALHO et al., 2011).

A flexibilidade foi avaliada pelo teste de Rikli e Jones (2008) de 'sentar e alcançar' que tem o objetivo de avaliar a flexibilidade dos membros inferiores. Para a aplicação do teste foram necessárias uma cadeira com encosto e sem braços, com uma altura aproximada de $43 \mathrm{~cm}$ do chão até o assento, e uma régua de $50 \mathrm{~cm}$. O dedo hálux é a referência do ponto zero (0) para a medida desse teste. Dessa forma, o registro terá um resultado negativo (-) quando a distância for anterior ao dedo hálux e, quando essa medida for além do dedo hálux, o registro será positivo (+).

A intervenção foi realizada no Laboratório de ER com Ênfase em Saúde (LERES-Tucuruí), no período da manhã. O ER foi realizado em um ambiente climatizado, com frequência de três sessões semanais, em dias alternados, com a duração total de uma hora, tendo início às 06h30min e término às 07h30min, no período de abril a outubro de 2014.

O modelo de periodização foi o linear. No início de cada sessão, as voluntarias foram submetidas a exercícios de alongamento estático, com 10 s de insistência, repetidos ao término de cada sessão. As voluntárias realizaram o teste de 1RM para estabelecer a intensidade das cargas correspondentes ao treinamento que foi planejado para três ciclos bimestrais:

a) No primeiro ciclo, a intensidade foi de $50 \%$ de $1 \mathrm{RM}$;

b) No segundo ciclo a intensidade foi de $60 \%$;

c) No terceiro e último ciclo a intensidade passou a ser de $70 \%$.

Cabe destacar que a cada mudança de ciclo era realizado um novo teste de 1RM. O método usado foi o alternado por segmento, com 3 séries de 12-15 repetições para o primeiro ciclo, 10-12 repetições para o segundo ciclo e 8-10 repetições para o terceiro ciclo, com intervalos de $30 \mathrm{~s}$ a $45 \mathrm{~s}$ entre as séries e de 30s a 1min entre os exercícios, seguindo as recomendações do ACSM (2014).

Inicialmente, realizou-se estatística descritiva para caracterizar a amostra; posteriormente, utilizou-se a análise de normalidade pelo teste de Shapiro-Wilk e, de acordo com os resultados, realizou-se a ANOVA one way com pós-hoc de Bonferroni para as análises intra e inter-testes para as variáveis de força muscular. Já para as outras variáveis utilizou-se o teste $t$ de Student para amostras pareadas. A diferença percentual foi calculada pela fórmula:

$$
\Delta \%=[(\text { Pós-teste-Teste }) * / \text { Teste }]
$$

O nível de significância adotado para o estudo foi de $\mathrm{p}<0,05$. O software utilizado para os cálculos foi o BioStat ${ }^{\circledR}$ versão 5.0. 


\section{Resultados}

A Tabela 1 mostra os dados relativos às características da amostra com medidas de tendência central e de dispersão:

Tabela 1 - Dados descritivos das voluntárias

\begin{tabular}{lcccc}
\hline \multicolumn{5}{c}{ Grupo de Treinamento $(\mathbf{n}=\mathbf{1 8})$} \\
Variáveis & Média & Desvio Padrão & Número Máximo & Número Mínimo \\
\hline Idade (anos) & 58,7 & 5,6 & 64,0 & 50,0 \\
Estatura (m) & 1,53 & 0,1 & 1,64 & 1,43 \\
Massa (Kg) & 69,0 & 14,5 & 98,4 & 57,9 \\
IMC $\left(\mathrm{Kg} / \mathrm{m}^{2}\right)$ & 29,2 & 4,2 & 36,6 & 24,3 \\
\hline
\end{tabular}

Fonte: Autoria própria (2015).

A Figura 1 mostra os resultados para a força muscular de membros superiores:

Figura 1 - Resultados para força de membros superiores com as comparações dos exercícios resistidos vs.

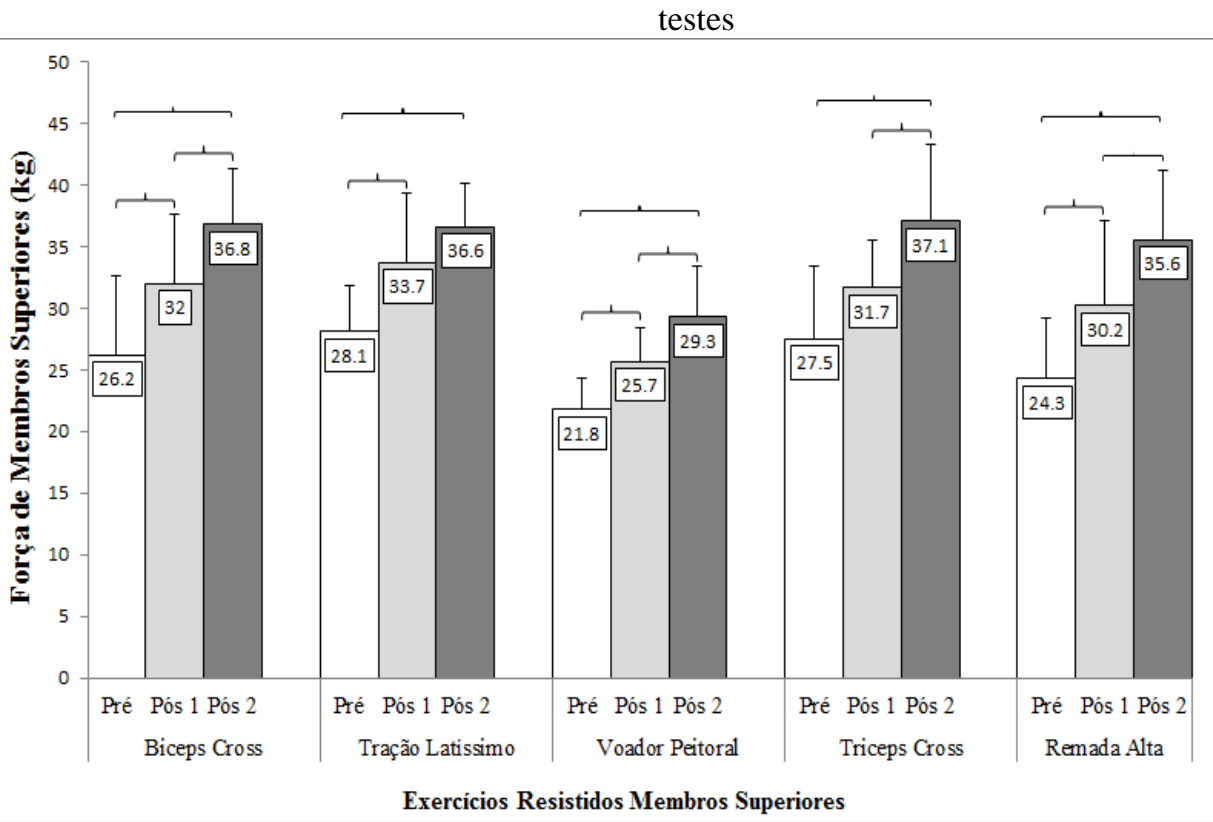

Obs.: O símbolo $\longrightarrow$ mostra diferença $\mathrm{p}<0,05$.

Fonte: Autoria própria (2015).

A ANOVA one way mostrou diferença estatística $(\mathrm{p}<0,05)$ para todos os ER deste seguimento corporal. O teste de Post-Hoc Bonferroni para as comparações múltiplas mostrou diferenças $(\mathrm{p}<0,05)$ para os seguintes exercícios vs. testes: bíceps cross apresentou melhoras em todos os testes (pré vs. pós $1: \Delta \%=22,1 \%$; pré vs pós $2: \Delta \%=40,5 \%$ e pós 1 vs. pós $2: \Delta \%=15 \%$ ); tração latíssimo apresentou melhoras no pós-teste $1(\Delta \%=19,9 \%)$ e $2(\Delta \%=30,2 \%)$ comparado ao pré-teste; voador apresentou melhoras em todos os testes (pré vs. pós $1: \Delta \%=17,9 \%$; pré vs pós 2 : $\Delta \%=34,4 \%$ e pós 1 vs. pós $2: \Delta \%=14 \%$ ); o tríceps cross apresentou melhoras no pós-teste 2 $(\Delta \%=34,9 \%)$ comparado ao pré-teste e no pós-teste $2(\Delta \%=17 \%)$ compara ao pós 1 ; e, finalmente a remada alta, que também apresentou melhoras em todos os testes (pré vs. pós $1: \Delta \%=24,3 \%$; pré vs pós $2: \Delta \%=46,5 \%$ e pós 1 vs. pós $2: \Delta \%=17,9 \%$ ).

A Figura 2 mostra os resultados para a força muscular de membros inferiores: 
Figura 2 - Resultados para força de membros inferiores com as comparações dos exercícios resistidos vs. testes

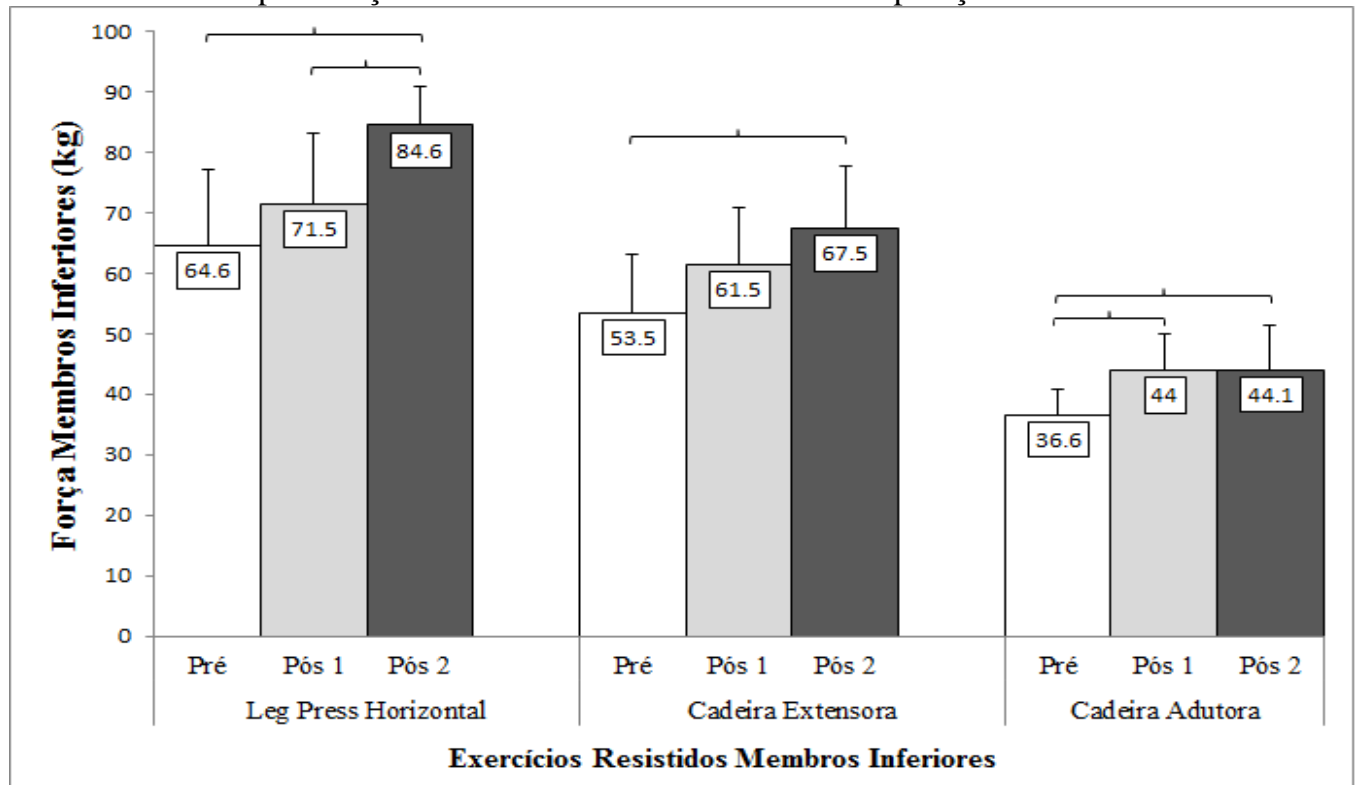

$\mathrm{O}$ símbolo $\longrightarrow$ mostra diferença $\mathrm{p}<0,05$.

Fonte: Autoria própria (2015).

A ANOVA one way mostrou diferença estatística $(\mathrm{p}<0,05)$ para os ER deste seguimento corporal. O teste de Post-Hoc Bonferroni para as comparações múltiplas mostrou diferenças ( $\mathrm{p}<0,05)$ para os seguintes exercícios vs. testes: leg press horizontal apresentou melhoras do pósteste $2(\Delta \%=31 \%)$ comparado ao pré-teste e pós-teste $2(\Delta \%=18,3 \%)$ comparado ao pós-teste 1 ; a cadeira adutora apresentou melhoras: (pré vs. pós $1: \Delta \%=20,2 \%$; pré vs pós $2: \Delta \%=20,5 \%$ ). Já para a cadeira extensora, o teste de Friedman mostrou melhora somente do pós-teste $2(\Delta \%=26,2 \%)$ comparado ao pré-teste.

A Figura 3 mostra os resultados para os testes do protocolo GDLAM:

Figura 3 - Resultados para os testes do protocolo GDLAM

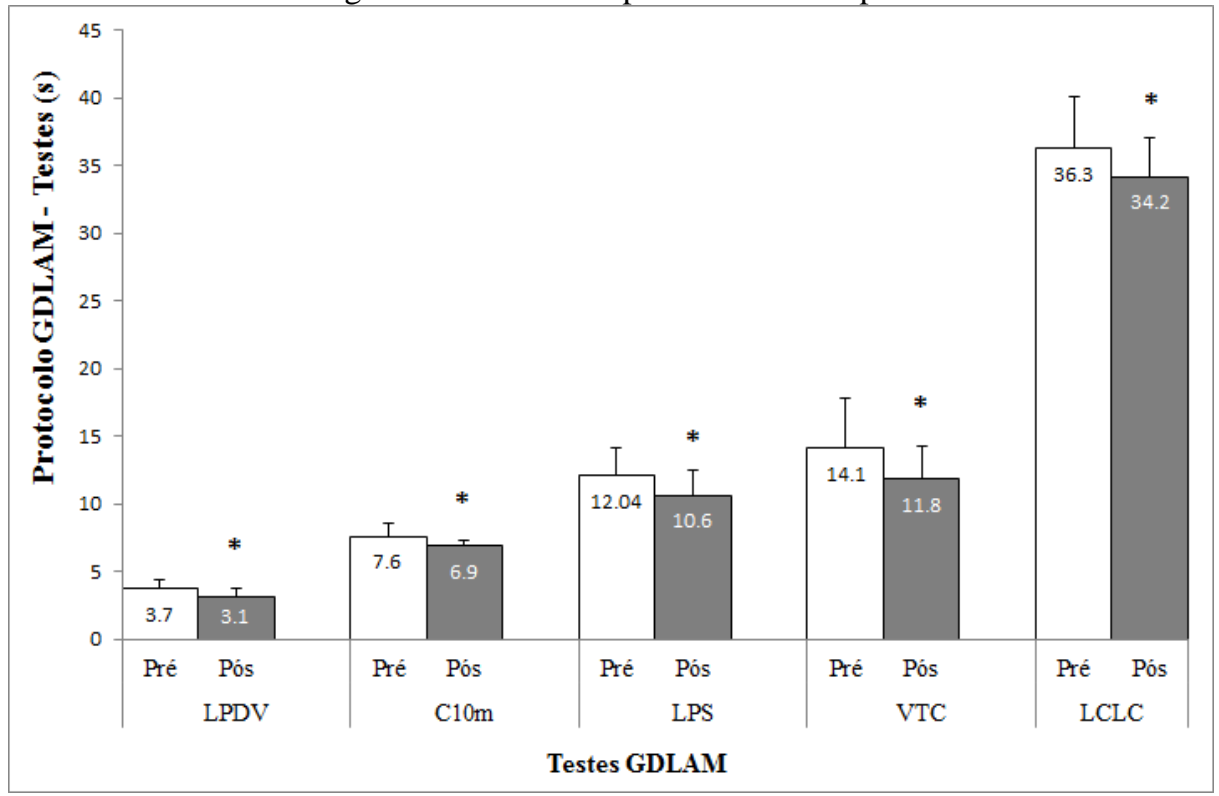

Obs.: O símbolo (*) mostra diferença $\mathrm{p}<0,05$.

Fonte: Autoria própria (2015).

$\mathrm{O}$ teste $T$ student mostrou diferença com melhora estatística $(\mathrm{p}<0,05)$ para todos os testes: $\operatorname{LPDV}(\Delta \%=-16,2 \%) ; \operatorname{C} 10 \mathrm{~m}(\Delta \%=-9,2 \%) ; \operatorname{LPS}(\Delta \%=-12 \%) ; \operatorname{VTC}(\Delta \%=-16,6 \%)$ e LCLC $(\Delta \%$ $=-5,8 \%)$. 
A Figura 4 mostra que escore do índice GDLAM (IG) também apresentou diferença com melhora estatística de $\mathrm{p}(\mathrm{p}=0,002 ; \Delta \%=-10,4 \%)$ comparado ao pré-teste.

Figura 4 - Resultados para o índice do protocolo GDLAM (IG)

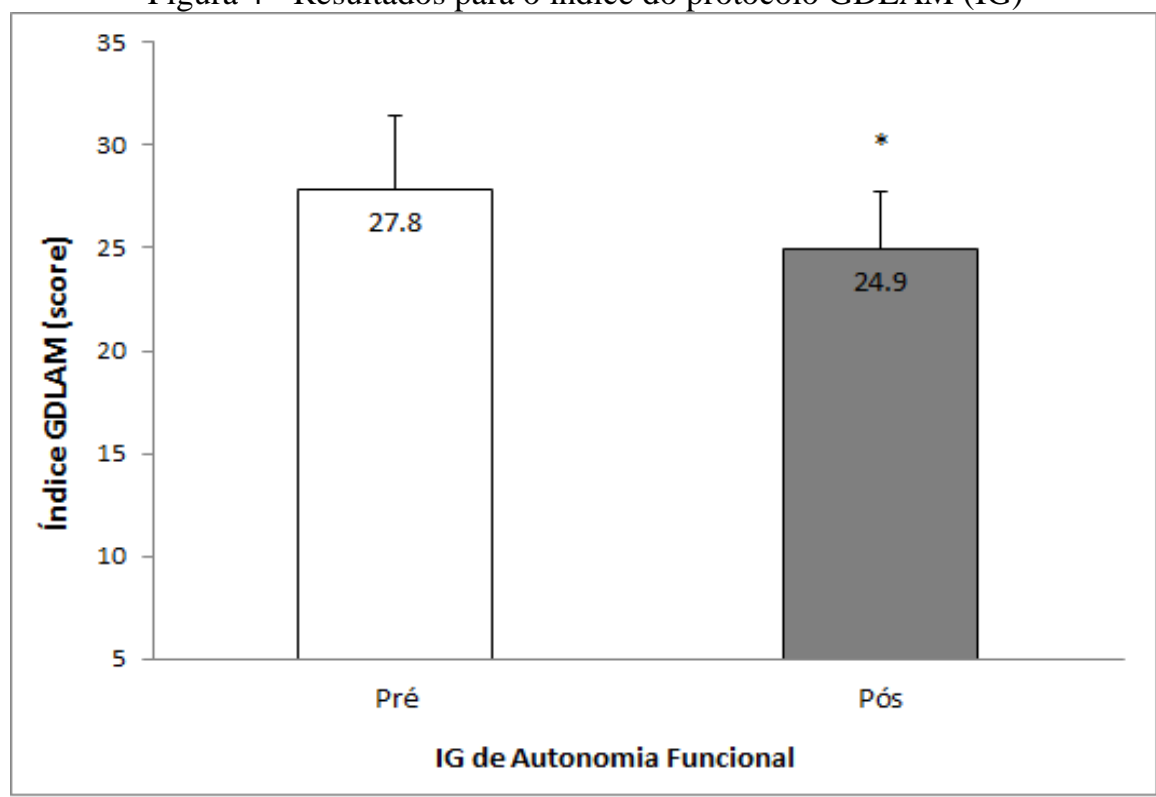

Obs.: O símbolo $(*)$ mostra diferença $\mathrm{p}<0,05$.

Fonte: Autoria própria (2015).

A Figura 5 mostra o resultado para o teste sentar e alcançar os pés que também presentou diferença $(\mathrm{p}<0,05)$, com melhora estatística de $\Delta \%=76.8 \%$ comparado ao pré-teste.

Figura 5 - Resultados para o teste de sentar e alcançar os pés

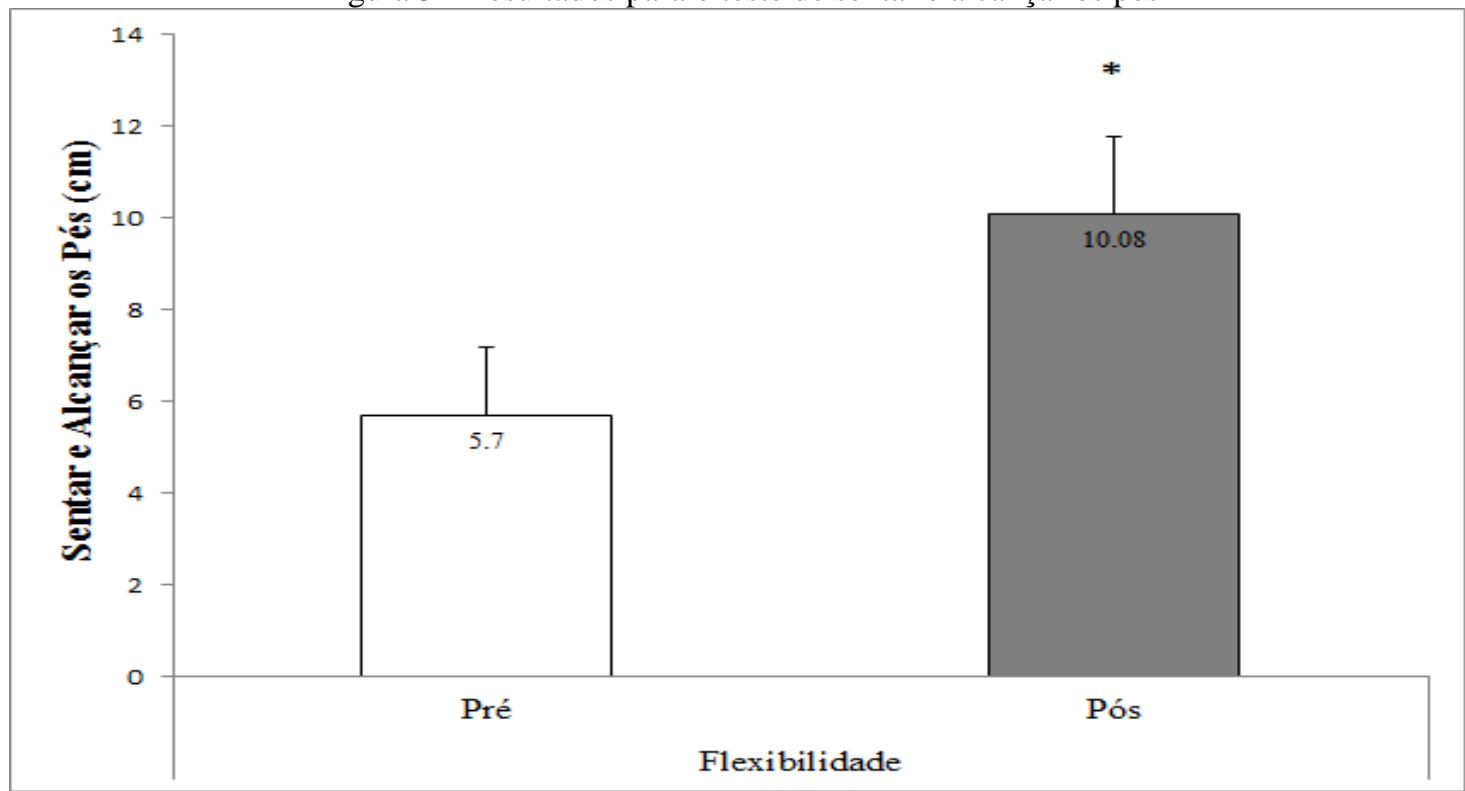

Obs.: O símbolo (*) mostra diferença $\mathrm{p}<0,05$.

Fonte: Autoria própria (2015).

A Figura 6 mostra os resultados para o protocolo de QV SF-36. Em relação ao pré-teste, houve melhora estatística $(\mathrm{p}<0,05)$ na saúde física $(\Delta \%=28,6 \%)$ e no escore total $(\Delta \%=17,9 \%)$. Entretanto, a saúde mental apresentou uma melhora $(\Delta \%=3,2 \%)$ não estatística $(p>0,05)$. 
Figura 6 - Resultados para o protocolo de QV SF-36

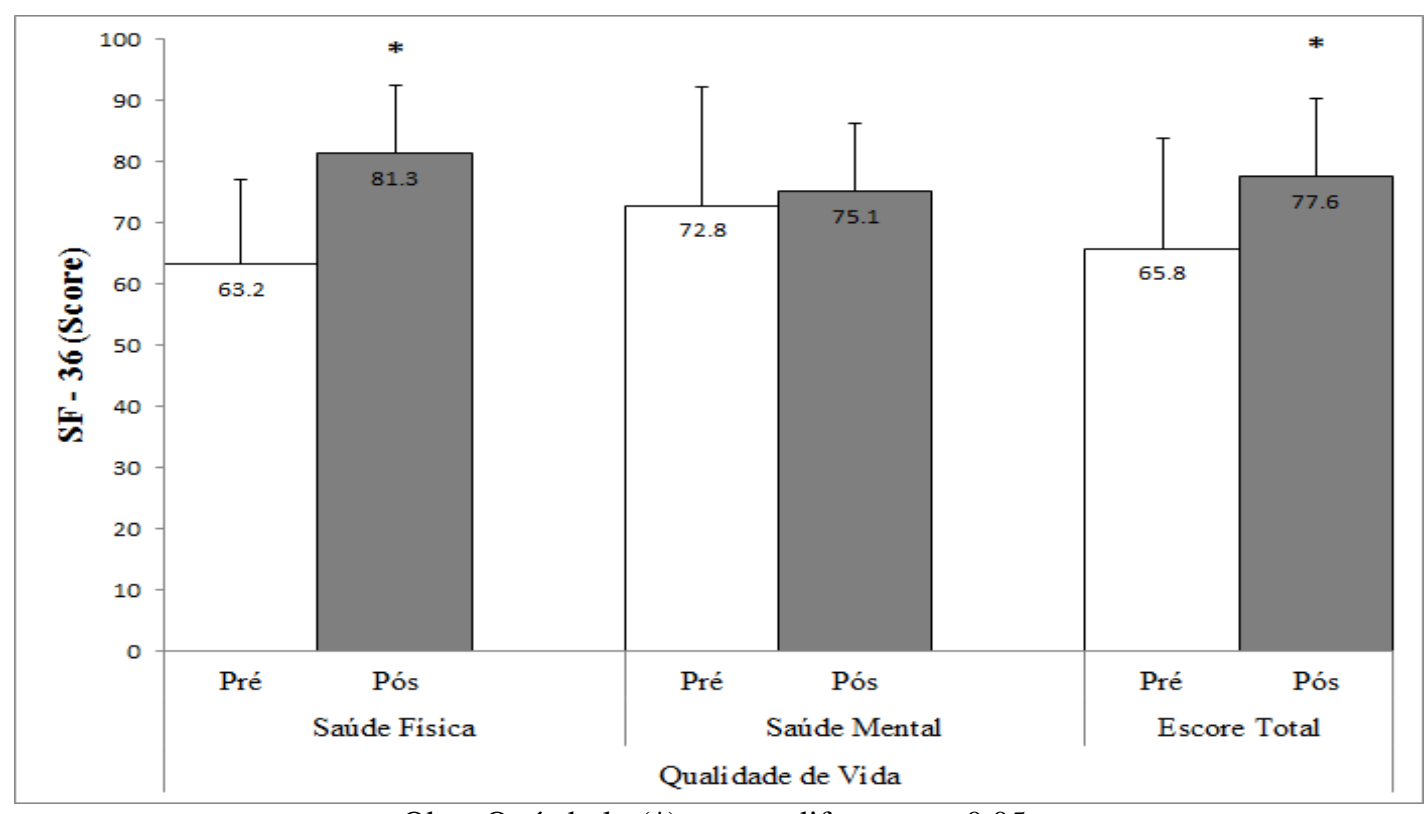

Obs.: O símbolo $(*)$ mostra diferença $\mathrm{p}<0,05$.

Fonte: Autoria própria (2015).

\section{Discussão}

Com o crescimento da população idosa e o aumento de sua expectativa de vida, o número de pesquisas abordando temas como o exercício físico, a saúde e a QV relacionada ao envelhecimento aumentaram significativamente (MATSUDO; MATSUDO; BARROS NETO, 2001; CORRÊA et al., 2013).

Acredita-se que o estilo de vida ativo é considerado fundamental para melhoria da QV, além de melhorar a saúde durante todo o processo de envelhecimento (CORREAA; BORBA-PINHEIRO; DANTAS, 2013). Os mesmos autores afirmam que a autonomia funcional é uma variável relevante no estudo da saúde, aptidão física e QV relacionada à saúde de pessoas mais velhas e idosas.

A autonomia é a capacidade de executar independente e satisfatoriamente as atividades do dia-a-dia, favorecendo as relações e as atividades sociais, bem como o exercício dos direitos e deveres dos cidadãos (ABREU et al., 2002). Por esses motivos, considera-se a autonomia funcional uma das principais perdas com o avançar da idade e que merecem atenção especial de profissionais de saúde (CORRÊA; BORBA-PINHEIRO; DANTAS, 2013).

Em um estudo que avaliou a capacidade funcional de 60 idosos, através do desempenho de atividades da vida diária, o grupo que praticou ER apresentou melhores resultados $(\mathrm{p}<0,05)$, comparado aos grupos que praticavam hidroginástica e sedentários para os testes: $80 \mathrm{~m}$ de caminhada, subir e descer escadas, levantar da cadeira e locomover-se e levantar do solo; o grupo de ER também foi melhor $(\mathrm{p}<0,05)$ que os sedentários para o teste de calçar meias (FRANÇA et al., 2014).

O presente estudo evidenciou melhoras estatísticas para autonomia funcional em todos os testes do protocolo GDLAM e também para o escore do índice GDLAM (IG) (Figuras 3 e 4). Os resultados obtidos nesta pesquisa corroboram com o que vem sendo demonstrado na literatura, como em um estudo realizado com mulheres que praticaram ER por 3 meses e obtiveram melhoras estatísticas para autonomia funcional nos testes VTC, C10m, LPS, LCLC e no IG (CABRAL et al., 2014) e outro que apresentou melhoras no teste LCLC e no IG, após um treinamento de 12 semanas (FARIAS et al., 2014). Em outra pesquisa, também realizada com mulheres em idade avançada que participaram de um programa de ER durante 16 semanas, constatou-se melhora significativa $(\mathrm{p}<0,05)$ no tempo dos testes de C10m e de LPS (VALE et al., 2006). Diante dos resultados 
discutidos, é possível constatar que a prática regular e orientada do ER pode favorecer a manutenção e o aumento dos níveis de autonomia funcional de mulheres em idade avançada.

A QV é subjetiva e sofre influência de vários fatores que afetam a saúde e, consequentemente, a vida do ser humano (MERQUIADES et al., 2009; VIEIRA et al., 2012). Entre os fatores que são afetados, no presente estudo, considera-se a condição física, psíquica e social (VIEIRA et al., 2012; CORRÊA; BORBA-PINHEIRO; DANTAS, 2013). Para Gaede-Carrillo et al. (2015), em um estudo com 66 idosos que praticam exercícios físicos regulares, pelo menos 50\% dos sujeitos apresentaram pontuação máxima (100 pontos) para os domínios referentes aos aspectos físicos, sociais e emocionais, com pontuação mediana acima de 70 pontos para outros aspectos, com exceção do domínio dor.

Os resultados para QV mostraram que o grupo que realizou ER linear apresentou melhora estatística $(\mathrm{p}<0,05)$ para a saúde física e o escore total. Vários estudos envolvendo diferentes exercícios também evidenciam melhoras na QV de pessoas mais velhas e idosas, como o que ao avaliar 15 idosos praticantes de hidroginástica, no período de seis meses, auferiu média do escore

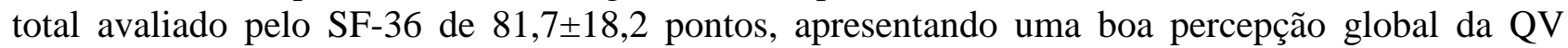
(PEREIRA; BORGES, 2011). Outro estudo que também utilizou o SF-36 para avaliar a QV de 36 idosos praticantes de algum tipo de atividade física por no mínimo dois anos, evidenciou que os escores gerais e todos os componentes se classificaram em uma boa $\mathrm{QV}$, sendo que o componente saúde mental apresentou os melhores resultados (SILVA et al., 2011), o que não ocorreu no presente estudo, pois a variável saúde mental não apresentou melhoras estatísticas. No entanto, Vieira et al. (2010), em pesquisa com 16 idosas que praticaram ER durante 8 semanas, 3 vezes por semana em dias alternados, evidenciou melhora significativa $(\mathrm{p}<0,05)$ nos domínios de estado geral de saúde e componente físico, o que corrobora com o presente estudo.

No que se refere à força muscular em indivíduos sedentários, é sabido que essa variável atinge um pico entre os 20 e 30 anos, com forte tendência a diminuição gradativa a partir dos 30 anos e nos 20 anos seguintes (REBELATTO et al., 2006). A redução de força e de massa muscular é chamada na literatura de sarcopenia (BAUMGARTNER; KOEHLER; GALLAGHER, 1998; MAIOR, 2004). As causas pelo desencadeamento do processo de sarcopenia ainda não são totalmente conhecidas, mas acredita-se que é um processo multifatorial que inclui diminuição dos níveis de hormônios e da síntese proteica e inatividade física, as quais contribuem fortemente para a potencialização deste processo (OLIVEIRA et al., 2008; PÍCOLI; FIGUEIREDO; PATRIZZI, 2011).

Cabe destacar que, partir da idade avançada, a perda de força torna-se mais grave, sendo responsável por vários problemas de ordem funcional para o individuo. Nesse sentido, o programa de ER se apresenta como um ótimo método para evitar as perdas de massa e de força muscular inerente ao envelhecimento, podendo melhorar, além da força, o equilíbrio para evitar quedas e fraturas, a QV, a autonomia e a independência funcional (TEIXEIRA; HESPANHOL; MARQUEZ, 2012; FARIAS et al., 2014). O estudo de Harries e Bassey (1990) reforçam essa discussão, pois mostrou que os indivíduos têm um declínio da força máxima de $15 \%$ entre os 60 e os 70 anos, por conseguinte, esse declínio passa a ser 30\% maior para cada década vivida após os 70 anos.

O presente método de ER apresentou-se como uma forma eficaz e potencial para manter e aumentar a força muscular dos indivíduos estudados e, com isso, controlar e/ou retardar a instalação efetiva do processo de sarcopenia de indivíduos em idade avançada. Os resultados apontam para uma melhora estatística na força máxima de todos os exercícios após seis meses de ER, com intensidade progressiva de 50-70\%, tanto para os membros superiores, quanto para os membros inferiores. Corroborando com o presente estudo, Farias et al. (2014), em uma intervenção com ER e funcionais em 12 semanas, observaram melhora na força de membros inferiores e superiores.

O American College of Sports Medicine (ACSM, 2014) preconiza a utilização de cargas acima de $65 \%$ de 1RM para obtenção de hipertrofia e de orça muscular, o que vem ao encontro com os resultados positivos da pesquisa quanto ao ganho de força dos indivíduos. Borba-Pinheiro et al. (2010), em pesquisa realizada com voluntárias na pós-menopausa em um programa de ER linear com duração de 12 meses e intensidades de 70 a $90 \%$ de $1 \mathrm{RM}$, constataram melhoras $(\mathrm{p}<0,05)$ em 
todas as medidas de força tanto para os membros superiores quanto para os membros inferiores, além de melhoras significativas $(\mathrm{p}<0,05)$ no equilíbrio corporal e na $\mathrm{QV}$, corroborando com os achados do estudo de Queiroz e Munaro (2012), onde foram analisados os efeitos de um programa de ER com duas sessões semanais. Neste, as intensidades progrediram de 50 a $70 \%$ de 1RM durante oito semanas sobre a força muscular e sobre a autopercepção de saúde das idosas, mostrando aumento significativo $(\mathrm{p}<0,05)$ na força, sendo a maior com os exercícios de extensão do cotovelo $(69,8 \%)$ e leg press $(69,5 \%)$.

Vital et al. (2011), ao avaliar 14 idosos de ambos os sexos, realizando ER por 8 semanas com 3 séries e intensidades de 60 a $80 \%$ de 1RM, também constataram melhora significativa de força no grupo de ER com incremento de até 50\% comparado ao grupo de controle. Os estudos mencionados e os resultados da presente pesquisa reforçam a constatação de Albino et al. (2012), o qual afirma que programas de ER de alta intensidade podem melhorar a força de idosos em até $227 \%$ de 1 RM, enquanto o treinamento de baixa intensidade, permite incremento $\leq$ a $20 \%$.

A flexibilidade declina com o avanço da idade devido à deficiência de colágeno e fatores associados como maior rigidez dos tendões, ligamentos e capsula articular. O declínio ameaça a independência funcional por restringir a amplitude de movimento das grandes articulações e impedir os idosos de executarem tarefas do cotidiano como utilizar o carro, subir uma pequena escada, pentear os cabelos e vestir-se (REBELATTO et al., 2006). Tais fatores determinam a necessidade de uma vida ativa, pois de acordo com estudo de Albino et al. (2012), os adultos perdem cerca de 8 a $10 \mathrm{~cm}$ de flexibilidade na região lombar e no quadril.

Os dados obtidos ao final desta pesquisa referentes à flexibilidade mostram diferença significativa $(\mathrm{p}<0,05)$ com melhora para o teste de sentar e alcançar os pés $(\Delta \%=76.8 \%)$. Esses resultados sugerem que as melhoras na autonomia funcional e na $\mathrm{QV}$, podem estar associadas com o aumento da flexibilidade.

Vale et al. (2006) desenvolveu um estudo que avaliou 22 voluntarias acima dos 60 anos, constatando melhora significativa nos níveis de flexibilidade no grupo submetido a intensidades de $75 \%-85 \%$ de 1RM por 16 semanas de ER. Outro estudo, de Lopes et al. (2011) com 12 sujeitos de ambos os sexos, com idade entre 55 e 65 anos, submetidos a 2 meses de ER com 3 sessões semanais, obteve melhora na flexibilidade de $21,6 \%$ no grupo submetido ao ER comparado ao controle, sendo utilizado o método alternado por segmento, o mesmo método usado nesta pesquisa. Os resultados da presente pesquisa evidencia a eficácia do ER linear na melhora dos níveis de flexibilidade em mulheres mais velhas.

Em estudos recentes, Gavi (2014) afirma que o treinamento de força pode ser tão bom quanto o treinamento de flexibilidade para melhorar esta última variável. O estudo de Gavi (2014) contou com 35 mulheres que realizaram ER duas vezes por semana com intensidade $45 \%$ de $1 \mathrm{RM}$ e outro grupo com 31 mulheres que fizeram exercício de flexibilidade duas vezes por semana por 16 semanas, utilizando protocolo que incluiu os principais grupos musculares e tendões. Ao final do estudo evidenciou-se ganho significativo $(\mathrm{p} \leq 0.05)$ de flexibilidade semelhante em ambos os grupos. Em outro estudo, Farias et al. (2014) mostram que um treinamento concorrente, que envolveu ER e funcional, também pode melhorar a flexibilidade, a força de membros inferiores e superiores, além da autonomia funcional de mulheres em idade avançada, vindo ao encontro dos achados da pesquisa hora apresentada.

Contudo, o ER pode ser um forte aliado no controle de variáveis relacionadas ao envelhecimento, tornando os indivíduos mais velhos e idosos com maior tempo de vida ativa, o que favorece a autonomia funcional e, consequentemente, a QV destes indivíduos.

\section{Considerações finais}

Conclui-se que o método de ER utilizado na pesquisa foi efetivo para melhorar a flexibilidade, a força muscular, a autonomia funcional e a QV de mulheres em idade avançada. 


\section{Referências}

ABREU, F. M. C.; DANTAS, E. H. M.; LEITE, W. O. D.; BAPTISTA, M. R.; ARAGÃO, J. C. B. Perfil da autonomia de um grupo de idosos institucionalizados. Revista Mineira de Educação Física, v. 10, n. 455, 2002.

ALBINO, I. L. R.; FREITAS, C. R.; TEIXEIRA, A. R.; GONÇALVES, A. K.; SANTOS, A. M. P. V.; BÓS, A. J. G. Influência do treinamento de força muscular e de flexibilidade articular sobre o equilíbrio corporal em idosas. Revista Brasileira de Geriatria e Gerontologia, v. 15, n. 1, p. 1725, 2012. Crossef

AMERICAN COLLEGE OF SPORT MEDICINE (ACSM). Diretrizes para testes de esforço e sua prescrição. 9. ed. Rio de Janeiro: Guanabara, 2014.

AMORIN, F. S.; DANTAS, E. H. M. Autonomia e resistência aeróbica em idosos. Fitness e Performance Journal, v. 1, n. 3, p. 47-59, 2002.

BAUMGARTNER, R. N.; KOEHLER, K. M.; GALLAGHER, D.; ROMERO, L.; HEYMSFIELD, S. B.; ROSS, R. R.; GARRY, P. J.; LINDEMAN, R. D. Epidemiology of sarcopenia among the elderly in New Mexico. American Journal Epidemiology, v. 147, n. 8, p. 755-763,1998. Crossef

BEZERRA, F. C.; ALMEIDA, M. I.; NOBREGA-THERRIEN, S. M. Estudos sobre envelhecimento no Brasil: revisão bibliográfica. Revista Brasileira de Geriatria e Gerontologia, v. 15, n. $1,2012$.

BLOCK, I. T.; GONÇALVES, K. K.; MEYER, M. T.; BENEDITO JÚNIOR, S.; LIBERALI, R. Análise da flexibilidade muscular em idosos. Revista Brasileira de Prescrição e Fisiologia do Exercício, v. 2, n. 7, p. 141-148, 2008.

BORBA-PINHEIRO, C. J.; CARVALHO, M. C. G. A.; SILVA, N. S. L.; BEZERRA, J. C. P.; DRIGO, A. J.; DANTAS, E. H. M. Efeitos do treinamento resistido sobre variáveis relacionadas com baixa densidade óssea de mulheres menopausadas tratadas com alendronato. Revista Brasileira de Medicina do Esporte, v. 16, n. 2, 2010. Crossef

BORBA-PINHEIRO, C. J.; CARVALHO, M. C. G. A.; SILVA, N. S. L.; DRIGO, A. J.; BEZERRA, J. C. P.; DANTAS, E. H. M. Bone density, balance and quality of life of postmenopausal women taking alendronate participating in different physical activity programs. Therapeutic Advances in Musculoskeletal Disease, v. 2, n. 4, p. 175-185, 2010. Crossef

BRASIL. Dados sobre o envelhecimento no Brasil. Brasília-DF: Secretaria de Direitos Humanos, 2013. Disponível em: http://www.sdh.gov.br/assuntos/pessoa-idosa/dadosestatisticos/DadossobreoenvelhecimentonoBrasil.pdf. Acesso em: 21 jan. 2015.

BRASIL. Comissão nacional de ética em pesquisa: resolução №196/96 de pesquisa com humanos. Brasília: Conselho Nacional de Saúde, 2012.

CABRAL, A. C. A.; MAGALHÃES, I. K. M.; BORBA-PINHEIRO, C. J.; ROCHA-JÚNIOR, O. R. M. B.; FIGUEIREDO, N. M. A.; DANTAS, E. H. M. Composição corporal e autonomia funcional de mulheres idosas após um programa de treinamento resistido. Revista de Pesquisa Cuidado é Fundamental Online, v. 6, n. 1, 2014. 
CICONELLI, R. M.; FERRAZ, M. B.; SANTOS, W.; MEINÃO, I.; QUARESMA, M. R. Tradução para a língua portuguesa e validação do questionário genérico de avaliação de qualidade de vida SF36 (BRASIL SF-36). Revista Brasileira de Reumatologia, v. 39, n. 3, 1999.

CORREA, D. G.; BORBA-PINHEIRO, C. J.; DANTAS, E. H. M. Qualidade de vida no envelhecimento humano. Praxia, v. 1, n. 1, 2013.

DANTAS, E. H. M.; FIGUEIRA, A. H.; EMYGDIO, R.; VALE, R. S. Functional autonomy GDLAM protocol classification pattern in elderly women. Indian Journal of Applied Research; v. 4 , n. 7 , p. 262-266, 2014. Crossef

FARIAS, C. M.; BORBA-PINHEIRO, C.; OLIVEIRA, M.; GOMES DE SOUZA, R. Efectos de un programa de entrenamiento concurrente sobre la fuerza muscular, flexibilidad y autonomía funcional de mujeres mayores. Revista Ciencias de la Actividad Física UCM, v. 15, n. 2, p. 13 $24,2014$.

FRANÇA, L.; SANCHES, T.; REGINA, T.; MADUREIRA, F.; COLANTONIO, E.;

BARTOLOTO, F.; SCORCINE, C. Variabilidade da frequência cardíaca e desempenho de AVDs de idosos treinados e sedentários. Revista Brasileira de Qualidade de Vida; v. 6, n. 1, p. 01-07, 2014. Crossef

GAEDE-CARRILlO, M. R. G.; REZENDE, C. P.; SEBASTIÃO, E. C. O.; SÓL, N. A. A. Avaliação da qualidade de vida relacionada à saúde de participantes do Programa Terceira Idade: Vitalidade e Cidadania. Revista Brasileira de Qualidade de Vida, v. 7, n. 1, p. 01-11, 2015. crossef

GAVI, M. B. R. O. Efeitos dos exercícios resistidos sobre a variabilidade da frequência cardíaca e os sintomas em pacientes com fibromialgia. 2014. 119 f. Tese (Doutorado em Ciências Fisiológicas) - Universidade Federal do Espírito Santo, Vitória, 2014.

HARRIES, J. M, BASSEY, E. J. Torque- velocity relationship for the knee stensors en women in their 3th na 7rd decades. European Journal of Applied Physiology, v. 60, p. 87-90, 1990. Crossef

LOPES, C. R.; VIANA, H. B.; MOTA, G. R.; VERLENGIA, R.; SILVA, E. C.; OLIVEIRA, F. B. Efeitos de um treinamento de força para pessoas com 55 anos e mais. Revista Kairós Gerontologia, v. 14, n. 6, p. 95-105, 2011.

MAIOR, A. S. Relação sarcopenia e treinamento de força. Revista de Fisioterapia UNICID, v. 3, n. 2, p. 125-1 39, 2004.

MATSUDO, S. M.; MATSUDO, V. K. R.; BARROS NETO, T. L. Atividade física envelhecimento: aspectos epidemiológicos. Revista Brasileira de Medicina do Esporte, v. 7, n. 1, p. 2-13, 2001.

MERQUIADES, J. C.; AGRA, J. H. M.; ALBUQUERQUE, K. M. D.; COSTA, R. C.; NAVARRO, A. P. A importância do exercício físico para á qualidade de vida dos idosos. Revista Brasileira de Prescrição e Fisiologia do Exercício, v. 3, n. 18, p. 597-614, 2009.

OLIVEIRA, R. J.; LIMA, R. M.; GENTIL, P.; SIMÕES, H. G.; ÁVILA, W. R. M.; SILVA, R. W.; SILVA, F. M. Respostas hormonais agudas a diferentes intensidades de exercícios resistidos em mulheres idosas. Revista Brasileira de Medicina do Esporte, v. 14, n. 4, 2008. rossef 
PEREIRA, E. S.; BORGES, I. S. análise da percepção geral de saúde e da qualidade de vida de idosos praticantes de hidroginástica a partir do instrumento SF-36. Revista Brasileira de Prescrição e Fisiologia do Exercício, v. 5, n. 27, p. 278-284, 2011.

PEREIRA JUNIOR, J. G.; ROCHA, C. A. Q. C.; TUCHER, G.; PAIXÃO J. A. Diferença na autonomia de idosos praticantes de diferentes programas de atividade física. Revista Científica Faminas, v. 6, n. 1, p. 1-11, 2010.

PÍCOLI, T. S.; FIGUEIREDO, L. L.; PATRIZZI, L. J. Sarcopenia e envelhecimento. Fisioterapia em Movimento, v. 24, n. 3, p. 455-462, 2011. Crossef

QUEIROZ, C. O.; MUNARO, H. L. R. Efeito do treinamento resistido sobre a força muscular e a autopercepção de saúde em idosas. Revista Brasileira de Geriatria e Gerontologia, v. 15, n. 3, p. 547-553, 2012. crossef

RAMALHO, G. H. R. O.; FILHO, M. L. M.; RODRIGUES, B. M.; VENTURINI, G. R. O.; SALGUEIRO, R. S.; PACE JÚNIOR, R. L.; MATOS, D. G. O teste de 1rm para predição da carga no treino de hipertrofia e sua relação com o número máximo de repetições executadas. Brazilian Jornal of Biomotricity, v. 5, n. 3, p. 168-174, 2011.

REBELATTO, J. R.; CALVO, J. I.; OREJUELA, J. R.; PORTILLO, J. C. Influência de um programa de atividade física de longa duração sobre a força muscular manual e a flexibilidade corporal de mulheres idosas. Revista Brasileira de Fisioterapia, v. 10, n. 1, p. 127-132, 2006. crossef

RIKLI, R. E.; JONES, J. C. Teste de aptidão física para idosos. São Paulo: Manole, 2008.

SILVA, A. C. F.; GONÇALVES, E.; MAGDALON, J.; PAIVA, L.; LIBERALI, R. Perfil da qualidade de vida de idosos praticantes de atividade física em uma academia no Rio de Janeiro. Revista Brasileira de Prescrição e Fisiologia do Exercício, v. 5, n. 26, p. 177-184, 2011.

SOL, N. A. A.; PRADO, I. B. H.; GAEDE-CARRILLO, M. R. G.; SERRA, C. O. P.; SEBASTIÃO, E. C. O.; SANTOS, M. A.; NIMER, M.; FIGUEIREDO, R. C. Programa terceira idade: vitalidade e cidadania - uma proposta de atenção interdisciplinar. Saúde Coletiva, v. 8, n. 54, p. 239-243, 2011.

TEIXEIRA, E. L.; HESPANHOL, K. C.; MARQUEZ, T. B. Efeito do treinamento resistido com oclusão vascular em idosas. Revista Brasileira de Prescrição e Fisiologia do Exercício, v. 6, n. 36, p. 560-568, 2012.

THOMAS, J. R., NELSON, J. K.; SILVERMAN, S. J. Métodos de pesquisa em educação física. 5. ed. Porto Alegre: Artmed, 2007.

VALE, R. G. S.; BARRETO, A. C. G.; NOVAES, J. S.; DANTAS, E. H. M. Efeitos do treinamento resistido na força máxima, na flexibilidade e na autonomia funcional de mulheres idosas. Revista Brasileira de Cineantropometia e Desempenho Humano, v. 8, n. 4, p. 52-58, 2006.

VIEIRA, R. H. G.; NOGUEIRA, I. D. B.; CUNHA, E. S.; FERREIRA, G. M. H.; NOGUEIRA, P. A. M. S. Influência do treinamento resistido na qualidade de vida de idosas com hipertensão arterial sistêmica. Revista Brasileira de Medicina do Esporte, v. 18, n. 1, 2012. rossef 
VIEIRA, R. H. G.; SILVEIRA, D. A. O.; CANUTO, P. M. D. C.; SOUZA, J. F.; CUNHA, E. S.; NOGUEIRA, I. D. B.; FERREIRA, G. M. H.; NOGUEIRA, P. A. M. S. Avaliação da qualidade de vida após treinamento resistido em idosas hipertensas. Revista Brasileira de Fisioterapia, v. 14, n. $1,2010$.

VITAL, T. M.; PATATAS, J. M.; TAVARES, G. H.; COELHO, A. P. G. M; COSTA, G. A.; NUNES, J. E. D.; PUGA, G. M. Efeito do treinamento resistido na força muscular e capacidade funcional em idosos ativos. Revista Digital, Buenos Aires, v. 16, n. 155, 2011.

WORLD HEALTH ORGANIZATION. Physical status: the use and interpretation of anthropometry. Report of a WHO Expert Comitee. Geneve: World Health Organization Technical Report Series, 1995. 